\title{
Independent Component Analysis based Denoising of Magnetic Resonance Images
}

\author{
Neelabh Sukhatme \\ Student, M.E, Control System, \\ Department of Electrical Engineering, \\ Jabalpur Engineering College, Jabalpur, (M.P)
}

\author{
Shailja Shukla, Ph.D \\ Professor \& Head, \\ Department of Computer Science \& Engineering, \\ Jabalpur Engineering College, Jabalpur, (M.P)
}

\begin{abstract}
Denoising is an essential step for most of the digital image processing systems. Image denoising involves the manipulation of the image data to produce a visually high quality image. MRI images are always corrupted by random noises. In denoising of magnetic resonance images it is very important to preserve the useful details rather than just increasing its peak signal to noise ratio (PSNR) value. Different noise models including additive and multiplicative types are used. They include Gaussian noise, salt and pepper noise, speckle noise. This paper uses Independent component analysis (ICA) for denoising of noisy MRI's. A comparative analysis was also performed, the output obtained by independent component analysis were compared with that obtained from discrete wavelet transform (DWT). The comparative analysis shows that the independent component analysis is better than the discrete wavelet transform in terms of peak signal to noise ratio (PSNR), Mean square error (MSE) and Mean structural similarity index metric (MSSIM).
\end{abstract}

\section{Keywords}

Discrete wavelet transform (DWT), Independent component analysis (ICA), Magnetic resonance imaging(MRI), Mean square error (MSE), Mean structural similarity index metric (MSSIM, Peak signal to noise ratio (PSNR).

\section{INTRODUCTION}

Images are considered as a powerful source of information and are extensively used in various fields. For example in medical industry, machine vision, space exploration etc. The field of image processing is thus considered to be very complex and of diverse nature. Due to this, image processing is one of the most challenging areas in mathematics, engineering, medical science, and entertainment industry. The advances in computer technology enabled us to process images formed by devices such as camera, scanner, ultrasounds, and X-rays to improve their quality, enhance their features, and combine different pieces of information. The digital image processing usually includes image restoration. Image restoration can be defined as a method of removal or reduction of degradation that are incurred during the image capturing. Images get degraded due to blurring as well as noise due to the electronic and photometric sources. The Blurring can be described as the form of bandwidth reduction of images which is caused because of imperfect image formation process such as relative motion between camera and original scene or by an optical system that is out of focus. Noises are those unwanted signals that interfere with the original signals and degrades the visual quality of digital image. The primary sources of noise in digital images are imperfect instruments, problem with data acquisition process, interference natural phenomena, transmission and compression [1].

Image denoising forms the preprocessing step in the field of digital image processing, medical science, research, technology and where somehow image has been degraded and needs to be restored before further processing. There are numerous types of images and these inherit different types of noises. Different noise models are used to present different noise types. The methods used for denoising the image tend to be problem specific and these methods depend upon the type of image and noise model. Various types of noises, noise models and denoising methods are discussed in this paper. The next section discusses the formation of magnetic resonance images, third section describes different noise models, fourth section describes denoising techniques, fifth section describes wavelet transform, sixth section describes ICA method, seventh section describes methodology used for denoising of images, eighth section shows the experimental results and ninth section concludes the paper followed by references.

\section{MAGNETIC RESONANCE IMAGING}

Magnetic Resonance Imaging (MRI) images are always corrupted by some kind of noises that are introduced during the process of obtaining the MRI images. To remove noise content from image has always been one of the standard problems of the image analysis. Thus denoising methods are always used to enhance the quality of the image Magnetic Resonance Imaging (MRI) is a distinguished medical imaging technique which is found to be very useful for assessment of the soft tissues in the body. MRI scanners use the phenomenon of nuclear spin resonance. Since the discovery of this imaging technique it has been used for many medical applications. Because of the resolution of MRI and the technology being essentially harmless it has emerged as the most accurate and desirable imaging technology [1]. Regardless of major improvements in recent years, magnetic resonance (MR) images often suffer from low signal-to-noise ratio (SNR), especially in cardiac and brain imaging. The paper is focused on denoising the brain MRI images, but the methods described here can be applied for denoising of any kind of images [2].

The clinical MRI data is normally corrupted by noises from the measurement processes. The automatic diagnosis systems designed are very sensitive to noise, so it necessary to remove these noise before using these image for automatic diagnosis. Noise reduction methods are in use to increase the signal to noise ratio and thereby improving the image quality. This paper uses independent component analysis technique to improve the quality of image [2]. 


\section{NOISE MODELS}

The noise models can be broadly classified into two types i.e. the noise present in image could be either in additive form or multiplicative form [2].

\subsection{Additive Noise Model}

This type of Noise signals are additive in nature they get added to the original signal to produce a corrupted noisy signal and follow the following model:

$$
\mathrm{I}_{\mathrm{n}}(\mathrm{x}, \mathrm{y})=\mathrm{I}_{\mathrm{o}}(\mathrm{x}, \mathrm{y})+\varepsilon(\mathrm{n})
$$

Where $\mathrm{I}_{\mathrm{o}}(\mathrm{x}, \mathrm{y})=$ Original image

$$
\varepsilon(\mathrm{n}) \quad=\text { Noise }
$$

The Gaussian noise is a type of additive noise it evenly distributes itself over the signal. This type of noise has a Gaussian distribution [3].

\subsection{Multiplicative Noise Model}

In the multiplicative noise model the noise signal gets multiplied to the original signal. The multiplicative noise model follows the following rule:

$$
\mathrm{I}_{\mathrm{n}}(\mathrm{x}, \mathrm{y})=\mathrm{I}_{\mathrm{o}}(\mathrm{x}, \mathrm{y})+n \times \mathrm{I}_{\mathrm{o}}(\mathrm{x}, \mathrm{y})
$$

Speckle noise is multiplicative noise. This type of noise occurs in almost all coherent systems such as SAR images, Ultrasound images etc. The source of this noise is random interference between the coherent returns. The speckle noise follows a gamma distribution [4].

\section{DENOISING TECHNIQUES}

The denoising techniques can be broadly classified into two type i.e. spatial domain filtering and transform domain filtering [5]. The application depends upon the type of image and noise present in the image. These methods are described as:

\subsection{Spatial domain filtering}

The spatial domain filtering methods are considered to be the traditional way to remove the noise from the digital images. These methods employ the spatial filters. Spatial domain filtering is further classified into linear filters and non-linear filters [5].

\subsubsection{Linear Filters}

A mean filter is the optimal linear for Gaussian noise in the sense of mean square error. Linear filters tend to blur sharp edges, destroy lines and other fine details of image. It includes Mean filter and Wiener filter.

\subsubsection{Mean Filter}

The mean filter acts on an image by smoothing the image. It reduces the intensity variations between the adjacent pixels. Mean filter is nothing just a simple sliding window spatial filter that replaces the centre value of the window with the average values of its all neighboring pixels values including itself. It is implemented with the convolution mask, which provides the results that is weighted sum of vales of a pixel and its neighbors.

\subsubsection{Weiner Filter}

In the Weiner filtering method it is required that the information about the spectra of noise and original signal is known. The wiener filter works well only if the underlying signal is smooth. Weiner filtering method implements the spatial smoothing and its model complexity control corresponds to the choosing the window size [5].

\subsubsection{Non- Linear}

The non linear filters tend to remove the noise without any attempts to explicitly identify it. Spatial filters employ a low pass filtering on the group of pixels with the assumption that noise occupies the higher region of frequency spectrum. Generally spatial filters remove the noise to reasonable extent but at the cost of blurring the images which in turn makes the edges in the picture invisible.

\subsubsection{Median Filter}

Median filter [7] follows the moving window principle and uses $3 \times 3,5 \times 5$ or $7 \times 7$ window. The median of window is calculated and the center pixel value of the window is replaced with that value [6].

\subsection{Transform domain filtering}

According to the choice of the "analysis function" [6], the transform domain filtering methods can be classified into the following two categories:

\subsubsection{Spatial-Frequency Filtering}

In the Spatial-Frequency Filtering the low pass filters using Fast Fourier Transform (FFT) are employed. For denoising an image a frequency domain filter is designed and then it is allowed to adapt to a cut-off frequency so as to distinguish the noise components from the useful signal in the frequency domain. These methods are time consuming and depend on the cut-off frequency and the filter function behavior. Furthermore, they may produce frequency artifacts in the processed image [10].

\subsubsection{Wavelet domain}

Usually noise is concentrated in the high frequency components of the signal, which correspond to small detail size when performing a wavelet analysis. Therefore, removing of some high frequency (small detail components), which may be distorted by noise, is a denoising process in the wavelet domain. The filtering operations in the wavelet domain can be categorized into wavelet thresholding; statistical wavelet coefficient model and undecimated wavelet domain transform based methods [7].

\section{WAVELET TRANSFORM}

During the past years, considerable research has been done on noise reduction. Depending on the noise model different algorithms are used. The most common type of noise which corrupts an Image is additive random Gaussian noise. There are many approaches to remove additive noise, such as average filters and mean filters. But the drawback with the linear filters is that they tend to blur sharp edges, destroy lines and other fine image details, and perform poorly in the presence of noise. Non-linear spatial filters employ a low pass filtering on groups of pixels with the assumption that the noise occupies the higher region of frequency spectrum. Low-pass filters will not only smooth away noise but also blur edges in images while the high-pass filters can make edges even sharper and improve the spatial resolution but will also amplify the noisy background. Using Wavelets for noise reduction have captured researcher's attention in image denoising due to their properties. Wavelets have capability of extracting detailed spatial-frequency information. This property gives a better discrimination between the noise and the real data. 
Wavelet means a "small wave". A wave is an oscillating function of time or space and is periodic, whereas wavelets are localized waves. Wavelets have their energy concentrated in time. In wavelet analysis the signal to be analyzed is multiplied with a wavelet function and then transform is computed for each segment generated.Wavelets are functions that are generated from the single function called the prototype or mother wavelet. These functions are generated by dilations (scaling) and translations (shifts) in time frequency domain. In wavelet analysis, we often speak of approximations and details. The approximations are the highscale, low-frequency components of the signal. The details are the low-scale, high-frequency components [7].

\section{INDEPENDENT COMPONENT ANALYSIS (ICA)}

Independent Component Analysis (ICA) is a statistical signal processing technique having emerging new practical application areas, such as blind separation of mixed voices or images analysis of several types of data or feature extraction. Typical algorithms for ICA use centering, whitening (usually with the Eigen value decomposition), and dimensionality reduction as pre-processing steps in order to simplify and reduce the complexity of the problem for the actual iterative algorithm. Whitening and dimension reduction can be achieved with principal component analysis or singular value decomposition. Whitening ensures that all dimensions are treated equally a priori before the algorithm is run [8]. A simple mathematical representation of ICA is as follows. The data is represented by the random vector $X=\left(X_{1} \ldots X_{n}\right)$ and the components as the random vector $S=\left(S_{1} \ldots S_{n}\right)$. The task is to transform the observed data $\mathrm{X}$, using a linear static transformation into maximally independent components $\mathrm{S}$. Given the model and realizations (samples) $\left(X_{1} \ldots X_{n}\right)$ of the random vector $\mathrm{X}$, the task is to estimate both the mixing matrix A and the sources S. This is done by calculating the demixing matrix and using a cost function which either maximizes the non gaussianity of the calculated independent components or minimizes the mutual information. In some cases, a priori knowledge of the probability distributions of the sources can also be used in the cost function. Before the denoising of magnetic resonance images by ICA there are two preprocessing steps that are required [8]. These steps are:

\subsection{Centering}

Centering is performed to simply the ICA algorithm. Centering means to subtract the mean vector from the basis vector to make the variable zero mean. To center $X$ we subtract the mean $\mathrm{m}=\mathrm{E}\{\mathrm{X}\}$ so that the variable $\mathrm{X}$ becomes zero mean. After estimating mixing matrix A from the centered data the estimation is completed by adding mean vector of $\mathrm{S}$ back to the centered estimates of S [9].

\subsection{Whitening}

Second step in pre-processing is to whiten the observed variables. Whitening means that the observed vector $\mathrm{X}$ is linearly transformed thus a new vector $\tilde{X}$ is obtained which is white i.e. its components are uncorrelated and their variances equal unity [9].

The most frequently used ICA-method for calculating demixing matrix is FastICA. It is explained in the following:

\subsection{FastICA}

The FastICA method is an iterative fixed-point algorithm that was developed by Hyvärinen et al [6]. It is an alternative to gradient-based methods for maximizing non-Gaussianity and shows fast convergence. This method can be used for optimizing different types of cost functions, such as kurtosis or negentropy. Contrary to gradient-based methods, the FastICA method does not have a learning rate or other adjustable parameters. This is a great advantage, because in general, a bad choice of learning rate may destroy convergence [10].

\section{METHODOLOGY}

In order to quantify the performance of denoising algorithms, a high quality MRI image is taken and some known noise is added to it. In this paper three types of noises were used first is the Gaussian noise second speckle noise and third was salt and pepper noise. The level of noise is decided by noise variance and five different values were chosen to test our method. The images were first denoised using discrete wavelet transform and then the same noisy images were denoised using independent component analysis. The denoised image obtained by both the method was compared with the high quality MRI image and two image quality parameters were calculated. First the performance of each algorithm is compared by computing Peak signal to noise ratio (PSNR). The PSNR values of the input and output images both were noted down with the change in noise variance levels [11]. The PSNR is defined as:

$$
\begin{aligned}
& \text { MSE }=\frac{1}{m n} \sum_{i=0}^{m-1} \sum_{j=0}^{n-1}[I(i, j)-K(i, j)]^{2} \\
& \text { PSNR }=20 \log _{10}\left(\frac{M A X_{I}^{2}}{M S E}\right)
\end{aligned}
$$

After this the mean structural similarity index was calculated which gives the similarity between two images the value of MSSIM was also noted down [12]. The MSSIM is calculated as:

$$
\begin{aligned}
& \operatorname{SSIM}(x, y)=\frac{\left(2 \mu_{x} \mu_{y}+c_{1)}\left(2 \sigma_{x y}+c_{2}\right)\right.}{\left(\mu_{x}^{2}+\mu_{y}^{2}+c_{1}\right)\left(\sigma_{x}^{2}+\sigma_{y}^{2}+c_{2}\right)} \\
& \operatorname{MSSIM}(X, Y)=\frac{1}{M} \sum_{j=1}^{M} \operatorname{SSIM}\left(x_{j}, y_{j}\right)
\end{aligned}
$$

The comparative analysis using these two image quality measures gives us the idea that which algorithm gives us a better output.

\section{EXPERIMENTAL RESULTS}

MRI images used in this section are T1 weighted MRI images. The images were corrupted by three type of noises Gaussian, speckle, salt \& pepper respectively. The noise variance ranges from 0.01 to 0.09 . The noise variance values is directly related to the quality of image as the value of noise increases the image becomes more and more corrupted and it becomes difficult to extract the original image. The noisy version was denoised first using discrete wavelet transform to give a baseline comparison [13] [14]. Then the Independent component analysis technique was used for removal of noise from MRI. The noisy image PSNR value and denoised image PSNR values for both the techniques were noted. The result of experiment for Gaussian noise removal is shown in Table I, Table II Shows the result for Speckle noise removal and in Table III the results for salt \&pepper noise removal are given. The Input PSNR values are obtained from the noisy MRI's and the Output PSNR values are obtained from the denoised MRI. Fig. 1 shows a comparison between input vs. output 
PSNR values. The input value was obtained from data corrupted by Gaussian noise and the output value was obtained after noise elimination. Fig. 2 shows change in output PSNR with increase in noise variance level. The process was repeated for speckle noise removal and Fig. 3, Fig. 4 shows the result. Fig. 5 and Fig.6 shows the result for salt \& pepper noise removal. Fig. 7 shows the denoised images obtained for all three types of noise.

Table 1. Experimental result for Gaussian noise removal

\begin{tabular}{|c|c|c|c|}
\hline Noise Level & $\begin{array}{c}\text { P.S.N.R } \\
\text { (Original) }\end{array}$ & $\begin{array}{c}\text { P.S.N.R } \\
\text { (Denoised) } \\
\text { DWT }\end{array}$ & $\begin{array}{c}\text { P.S.N.R } \\
\text { (Denoised) } \\
\text { ICA }\end{array}$ \\
\hline 0.01 & 20.71 & 23.51 & 26.74 \\
\hline 0.03 & 16.33 & 21.83 & 22.18 \\
\hline 0.05 & 14.45 & 20.86 & 19.95 \\
\hline 0.07 & 13.16 & 20.01 & 18.49 \\
\hline 0.09 & 12.29 & 19.27 & 17.27 \\
\hline
\end{tabular}

Table 2. Experimental result for Speckle noise removal

\begin{tabular}{|c|c|c|c|}
\hline Noise Level & $\begin{array}{c}\text { P.S.N.R } \\
\text { (Original) }\end{array}$ & $\begin{array}{c}\text { P.S.N.R } \\
\text { (Denoised) } \\
\text { DWT }\end{array}$ & $\begin{array}{c}\text { P.S.N.R } \\
\text { (Denoised) } \\
\text { ICA }\end{array}$ \\
\hline 0.01 & 29.55 & 27.22 & 26.83 \\
\hline 0.03 & 24.90 & 25.88 & 26.02 \\
\hline 0.05 & 22.65 & 24.97 & 25.08 \\
\hline 0.07 & 21.31 & 24.45 & 24.65 \\
\hline 0.09 & 20.24 & 23.96 & 24.32 \\
\hline
\end{tabular}

Table 3. Experimental result for $S \& P$ noise removal

\begin{tabular}{|c|c|c|c|}
\hline Noise Level & $\begin{array}{c}\text { P.S.N.R } \\
\text { (Original) }\end{array}$ & $\begin{array}{c}\text { P.S.N.R } \\
\text { (Denoised) } \\
\text { DWT }\end{array}$ & $\begin{array}{c}\text { P.S.N.R } \\
\text { (Denoised) } \\
\text { ICA }\end{array}$ \\
\hline 0.01 & 24.53 & 25.36 & 27.01 \\
\hline 0.03 & 19.77 & 22.21 & 23.82 \\
\hline 0.05 & 17.69 & 20.87 & 21.81 \\
\hline 0.07 & 16.24 & 19.92 & 20.86 \\
\hline 0.09 & 15.01 & 19.24 & 19.33 \\
\hline
\end{tabular}

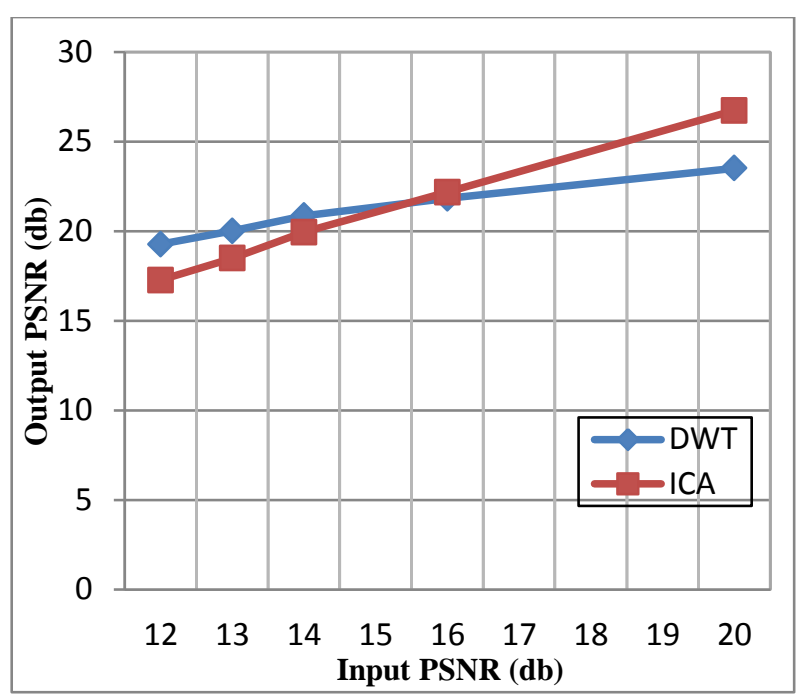

Fig 1: Input vs Output PSNR comparison result for image corrupted by Gaussian noise

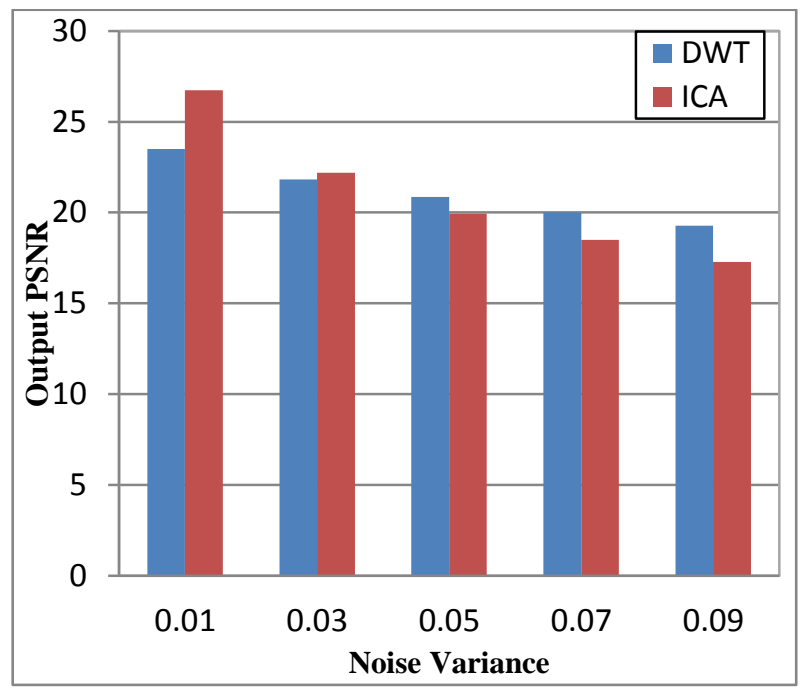

Fig 2: Noise variance vs Output PSNR values after Gaussian noise removal



Fig 3: Input vs Output PSNR comparison result for image corrupted by Speckle noise 


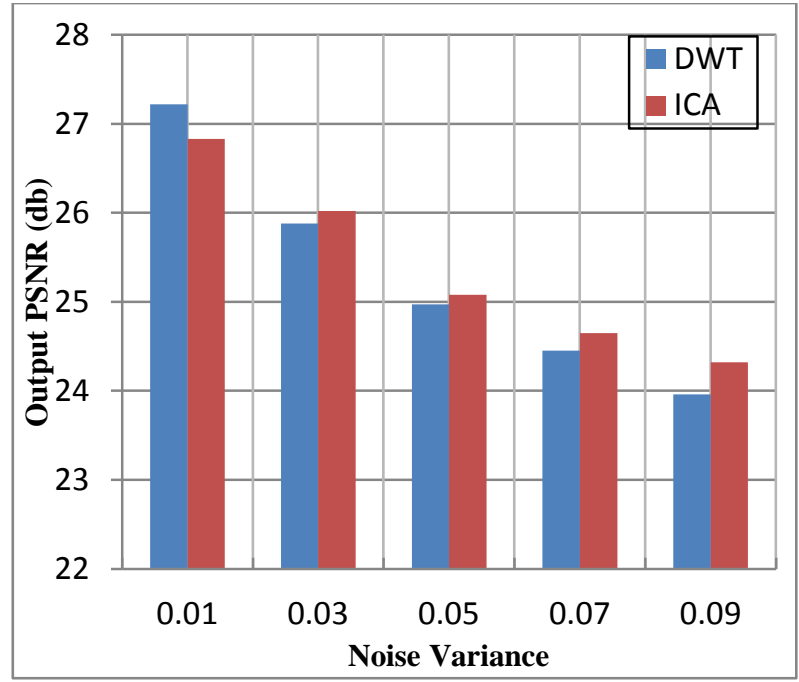

Fig 4: Noise variance vs Output PSNR values after Speckle noise removal

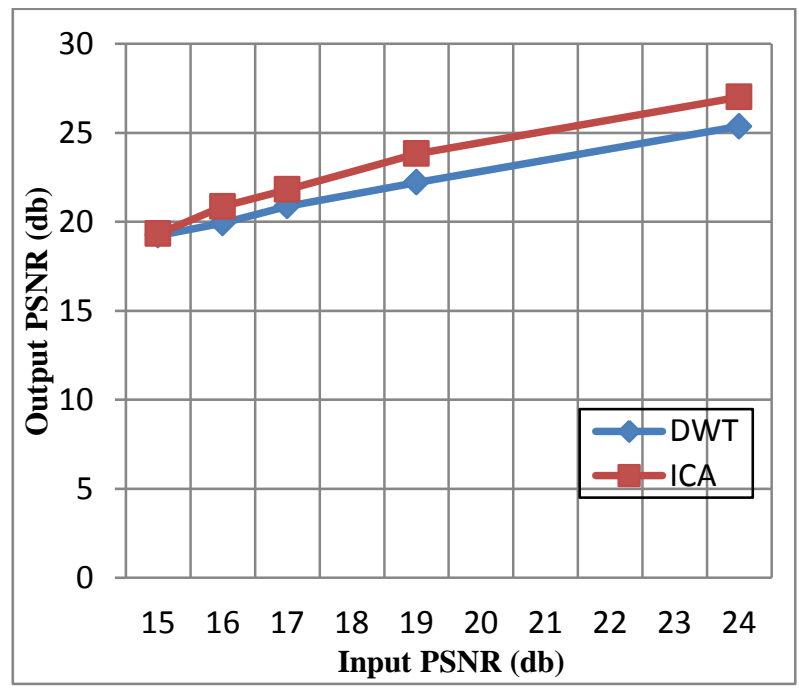

Fig 5: Input vs Output PSNR comparison result for image corrupted by Salt \& Pepper noise

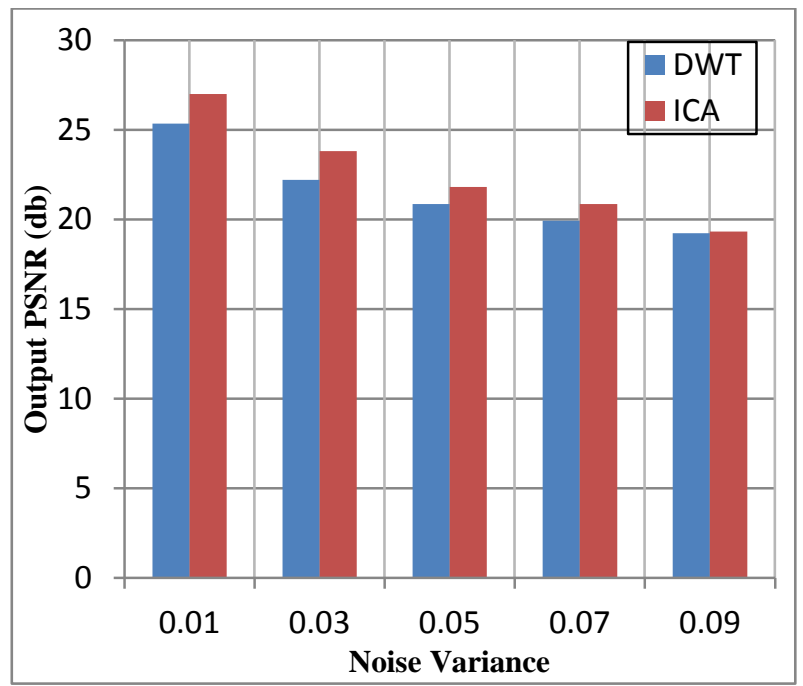

Fig 6: Noise variance vs Output PSNR values after Salt \& Pepper noise removal

\section{CONCLUSION}

This paper uses Independent component analysis for denoising of magnetic resonance images. A comparative analysis was performed between two techniques, Independent component analysis (ICA) and discrete wavelet transform (DWT). The results obtained are compared on the basis of PSNR values obtained from the experiments performed on the noisy images. The ICA method was found to be the best for removal of all three types of noise. The results obtained from the denoising experiments presented would be tried to be improved further by using ICA with other transform domain methods.

\section{REFERENCES}

[1] G.A. Wright, "Magnetic Resonance Imaging", IEEE Signal Processing Magazine, vol.1, pp. 56-66, 1997.

[2] A. Macovski,"Noise in MRI”, Magnetic Resonance Med, vol. 36, pp. 494-497, 1996.

[3] A. K. Jain. Fundamentals of digital image processing. Prentice Hall, Englewood Clis, NJ, c1989.

[4] E. R. McVeigh, R. M. Henkelman, and M. J. Bronskill, "Noise and filtration in magnetic resonance imaging", Med. Phys., vol. 3, pp. 604-618, 1985.

[5] M. C. Motwani, M. C. Gadiya, R. C. Motwani, and Jr. F. C. Harris. Survey of image denoising techniques. In Proceedings of Global Signal Processing Expo and Conference (GSPx 04), Santa Clara, CA, USA, September 2004.

[6] H. G. Stark. Wavelets and Signal Processing: An Application-Based Introduction. Springer, Berlin, Heidelberg, 2005.

[7] Tinku Acharya and Ajoy K. Ray, "Image ProcessingPrinciples and Appilications", Hoboken, New Jersey, A John Wiley \& Sons, Mc. Publication, 2005.

[8] Hyvarinen, J. Karhunen, and E. Oja," Independent Component Analysis," John Wiley \& Sons, New York, 2001.

[9] Balika S.Tawade, Rupali S. Kamathe, "MRI Image Denoising by the ICA \& PCA", Advance Image Processing, 2011.

[10] Hyvärinen, A., Hoyer, P., Oja, E.: "Image Denoising by Sparse Code Shrinkage”.In: Hykin, S., Kosko, B. (eds): Intelligent Signal Processing, IEEE Press, 2001.

[11] Y. Fisher, Fractal Image Compression - Theory and Application Springer-Verlag, New York, 1995.

[12] Detail information about structural similarity index from www.en.wikipedia.org/wiki/Structural_similarity.

[13] Sachin D Ruikar, Dharmpal D Doye,"Wavelet Based Image Denoising Technique", IJACSA,Vol. 2, No.3, pp 49-53 March 2011.

[14] M. N. Do, M. Vetterli, "The contourlet transform: an efficient directional multiresolution image representation,'IEEE Trans. on Image Process., vol. 14, no. 12, pp.2091-2106, 2005. 


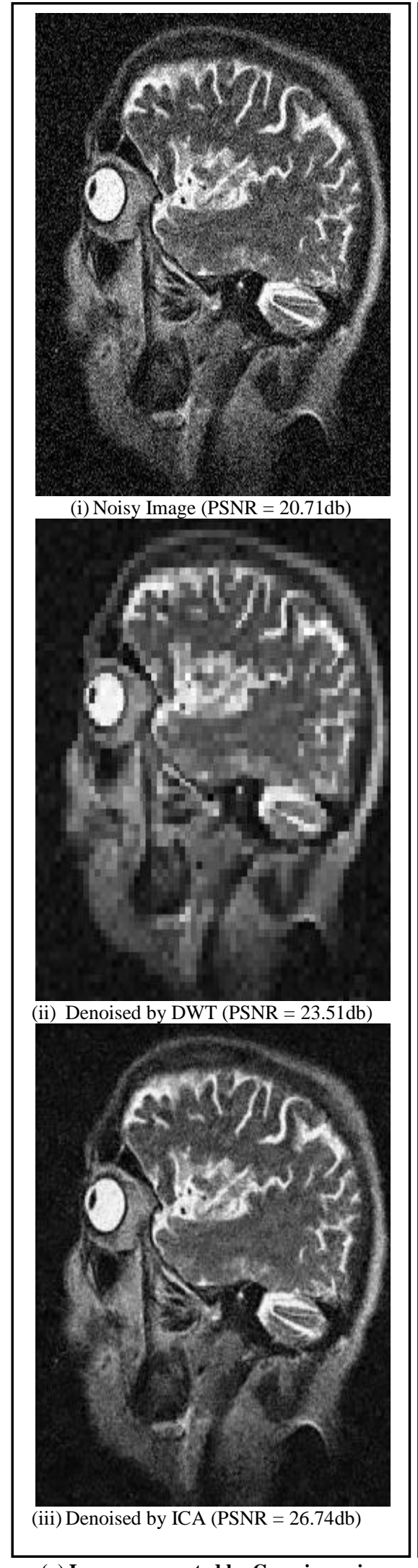

(a) Images corrupted by Gaussian noise

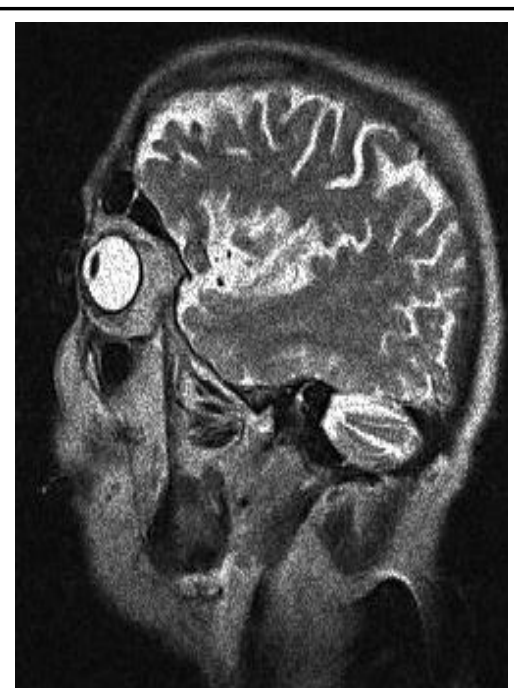

(i) Noisy Image (PSNR $=29.55 \mathrm{db})$

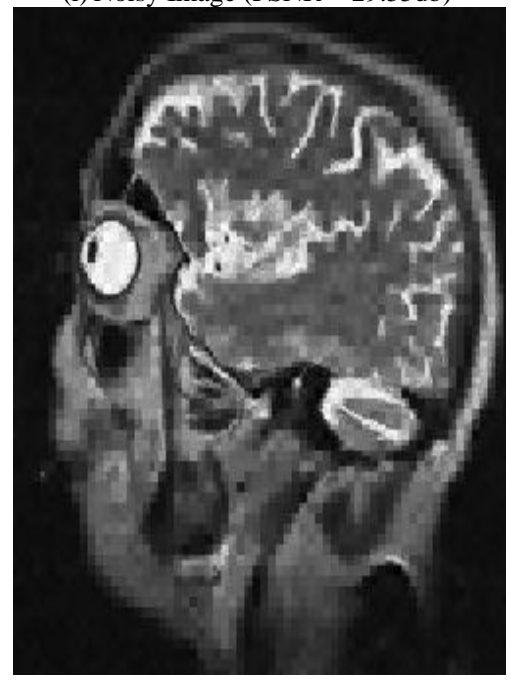

(ii) Denoised by DWT (PSNR $=27.22 \mathrm{db})$

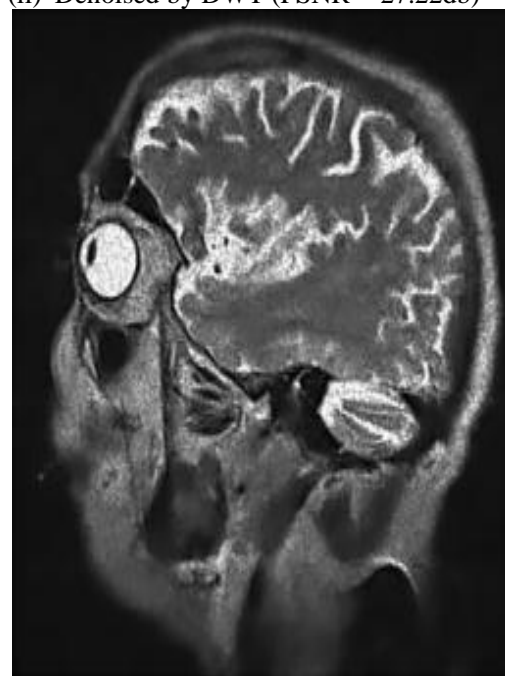

(iii) Denoised by ICA (PSNR $=26.83 \mathrm{db})$

(b) Images corrupted by Speckle noise

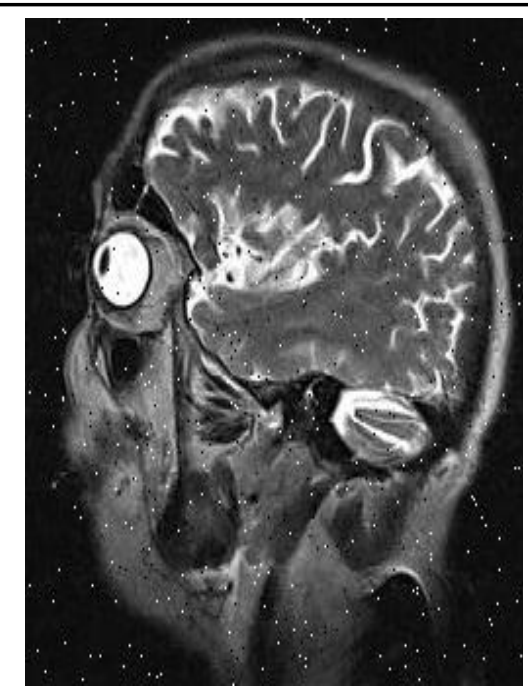

(i) Noisy Image (PSNR $=24.53 \mathrm{db})$

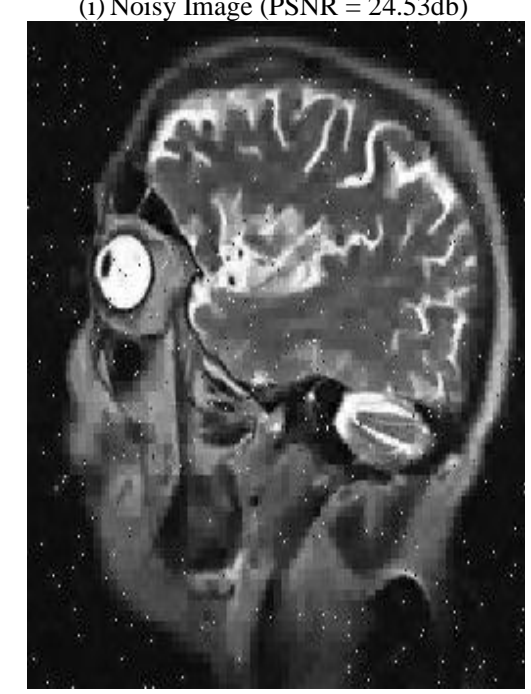

(ii) Denoised by DWT (PSNR $=25.36 \mathrm{db})$

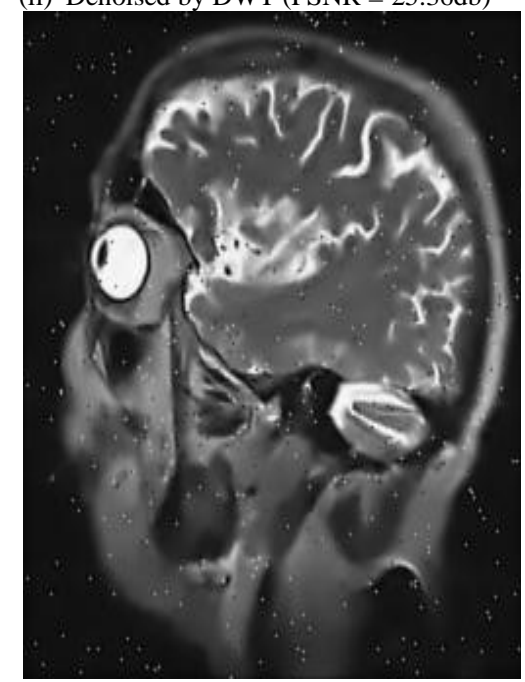

(iii) Denoised by ICA (PSNR $=27.01 \mathrm{db})$

(c) Images corrupted by Salt \& pepper noise

Fig 7: Denoising Results for removal of three types of noise (a) Gaussian, (b) Speckle, (c) Salt \& pepper 